\title{
Anisotropic hot-electron kinetics revealed by terahertz fluctuation
}

Le Yang ${ }^{1,2, \dagger}$, Zhenghua $\mathrm{An}^{2,3,6, \dagger, *}$, Ruijie Qian ${ }^{2}$, Hong Pan ${ }^{2}$, Pingping Chen ${ }^{1}$, Wei $\mathrm{Lu}^{1,4, *}$ and Susumu Komiyama ${ }^{1,5,7}$

${ }^{1}$ National Laboratory for Infrared Physics, Shanghai Institute of Technical Physics, The Chinese Academy of Sciences, Shanghai 200083, China.

${ }^{2}$ State Key Laboratory of Surface Physics, Institute for Nanoelectronic Devices and Quantum Computing and Department of Physics, Fudan University, Shanghai 200433, China.

${ }^{3}$ Shanghai Qi Zhi Institute, 41th Floor, AI Tower, No. 701 Yunjin Road, Xuhui District, Shanghai, 200232, China.

${ }^{4}$ School of Physical Science and Technology, ShanghaiTech University, 393 Middle Huaxia Road, Pudong, Shanghai, 201210, China

${ }^{5}$ Department of Basic Science, The University of Tokyo, Komaba3-8-1, Meguro-ku, Tokyo, 153-8902, Japan.

${ }^{6}$ Zhangjiang Fudan International Innovation Center, Fudan University, Shanghai 201210, China.

7 Terahertz Technology Research Center, National Institute of Information and Communications Technology, Nukui- Kitamachi 4-2-1, Koganei, Tokyo 184-8795, Japan.

$\dagger$ These authors contribute equally to this work.

*Correspondence to: Corresponding authors: Z. A. (email: anzhenghua@ fudan.edu.cn); W.Lu (e-mail: luwei@shanghaitech.edu.cn).

\section{Supplementary information}




\section{Supplementary Text}

section S1. Dependence on the crystallographic orientation......................2

section S2. Dependence on the current polarity and characteristic transport lengths...............................................

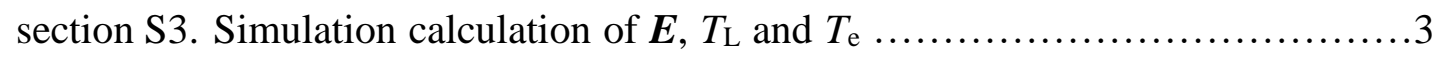

section S4. Hot Electron Domain (HED): High-electric-field domain or high-current filament? ................................................ 3

section S5. $\Gamma \rightarrow \mathrm{L}$ intervalley transfer of hot electrons in GaAs ...................4

section S6. Derivation of $T_{\mathrm{e}}$ in SNoiM................................. 4

section S7. Physical implication of $T_{\mathrm{e}}$ and intervalley noise................... 5

\section{Figures}

fig. S1 GaAs/AlGaAs quantum well structure................................ 7

fig. S2 Crystallographic-orientation dependence: $T_{\mathrm{e}}$-profile at $\mathrm{V}_{\mathrm{b}}=+10 \mathrm{~V} \ldots \ldots \ldots \ldots . .8$

fig. S3 Bias-polarity dependence: $T_{\mathrm{e}}$-image in device $\mathrm{D} 1_{[110]}$ for $\mathrm{V}_{\mathrm{b}}= \pm 10 \mathrm{~V} \ldots \ldots \ldots . . .9$

fig. S4 Physical model structure for simulation................................10

fig. S5 Simulation calculation of the electric field $\boldsymbol{E}$ for $V_{\mathrm{b}}=10.0 \mathrm{~V} \ldots \ldots \ldots \ldots \ldots . .11$

fig. S6 Simulation calculation: $T_{\mathrm{L}}$ and $T_{\mathrm{e}}$ profiles for $V_{b}=10 \mathrm{~V} \ldots \ldots \ldots \ldots \ldots \ldots . \ldots 12$

fig. S7 Comparison between experiments and simulation........................13

References $(54-58)$...................................................... 14 


\section{Supplementary Text}

S1. Dependence on the crystallographic orientation

Additional experiments made on $\mathrm{D} 1_{[110]}, \mathrm{D} 2_{[100]}, \mathrm{D} 3_{[110]}$ and $\mathrm{D} 3_{[100]}$ strongly indicate that the $T_{\mathrm{e}}$-profile systematically depends on the crystallographic orientation of the $\mathrm{N}$-shaped channel as shown in fig. S2. At $V_{\mathrm{b}}=10 \mathrm{~V}$, the two hot spots at the corners develop to converge, forming a remarkably prolonged HED in devices $\mathrm{D} 1_{[110]}$ and D3 ${ }_{[110]}$, while they remain separated without being bridged in D2 ${ }_{[100]}$ and $\mathrm{D}_{[100]}$.

\section{S2. Dependence on the current polarity and characteristic transport lengths}

Bias polarity dependence of the phenomena has been studied for all the devices. No substantial change is discerned upon the polarity reversal in the observed structures of $T_{\mathrm{L}^{-}}$and $T_{\mathrm{e}}$-profiles as demonstrated in fig. $\mathrm{S} 3$ for the $T_{\mathrm{e}}$ images in device $\mathrm{D} 1_{[110]}$ taken with opposite bias voltages, $V_{b}= \pm 10 \mathrm{~V}$. The absence of current polarity dependence is consistent with the fact that the device size as well as the typical length scales of the observed of hot-electron structure, $L_{\text {structure }}=0.3 \sim 2 \mu \mathrm{m}$, are distinctly larger than the characteristic length scale of transport.

First, the mean free path of electrons, $l=v_{\mathrm{F}} \tau=55 \mathrm{~nm}$, is much smaller than $L_{\text {structure. }}$.

Here the momentum relaxation time, $\tau=\mathrm{m} \Gamma \mu / \mathrm{e}=75 \mathrm{fs}$, derived from the Hall mobility

$\left(\mu=0.167 \mathrm{~m}^{2} / \mathrm{Vs}\right)$ at $300 \mathrm{~K}$, and the Fermi velocity $\mathrm{v}_{\mathrm{F}}=\left(2 \mathrm{E}_{\mathrm{F}} / \mathrm{m}^{\Gamma}\right)^{1 / 2}=7.3 \times 10^{5} \mathrm{~m} / \mathrm{s}$ derived from the Fermi energy, $\mathrm{E}_{\mathrm{F}}=119 \mathrm{meV}$ of electrons with the effective mass $\mathrm{m}^{\Gamma}=$ $0.067 \mathrm{~m}_{0}\left(\mathrm{~m}_{0}\right.$ : the free electron mass) and the density $\mathrm{n}=3.3 \times 10^{24} \mathrm{~m}^{-3}$ in the QW. (The thermal velocity, $\mathrm{v}_{\mathrm{T}}=\left(3 \mathrm{k}_{\mathrm{B}} \mathrm{T} / \mathrm{m}^{\Gamma}\right)^{1 / 2}=4.2 \times 10^{5} \mathrm{~m} / \mathrm{s}$ at $\mathrm{T}=300 \mathrm{~K}$, is smaller than $\mathrm{vF}_{\mathrm{F}}$ ) The electron system is regarded as a semi-classical quasi two-dimensional electron gas (2DEG) because the width of the $\mathrm{QW}, \mathrm{W}_{\mathrm{QW}}=35 \mathrm{~nm}$, is shorter than the mean free path, $l=55 \mathrm{~nm}$, but is larger than the dephasing length $l_{\varphi}$.

Secondly, the average distance that electrons drift during the momentum relaxation time under the influence of electric field, $l_{\mathrm{d}}=v_{\mathrm{d}} \tau=1.3 \mathrm{~nm}$, is far smaller than $L_{\text {structure, }}$, where the drift velocity in the condition of $V_{\mathrm{b}}=10 \mathrm{~V}$ is estimated to be $v_{\mathrm{d}}=$ $j_{2 \mathrm{D}} /(\mathrm{e} n) \approx 1.7 \times 10^{4} \mathrm{~m} / \mathrm{s}$ from the experiment $\left(j_{2 \mathrm{D}}=I / \mathrm{W}=0.6 \mathrm{~mA} / 2 \mu \mathrm{m}\right.$ in Fig. $\left.1 \mathrm{C}\right)$, and the momentum relaxation time $\tau \approx 75$ fs is nearly unchanged with increasing $\boldsymbol{E}$.

Thirdly, the average distance of electron drift during the electron-phonon energy relaxation time $\tau_{\mathrm{e}-\mathrm{ph}}$ under the influence of electric field, $l_{\mathrm{d} \text {, e-ph }}=v_{\mathrm{d}} \tau_{\mathrm{e}-\mathrm{ph}}$, is roughly estimated to be $l_{\mathrm{d} \text {, e-ph }} \sim 14 \mathrm{~nm}$, which is far smaller than $L_{\text {structure. }}$ Here, $\tau_{\mathrm{e} \text {-ph }} \approx 3 \mathrm{x}$ $\tau_{\mathrm{LO}, \mathrm{HD}} \approx 0.8 \mathrm{ps}$ is estimated from the emission time, $\tau_{\mathrm{LO}, \mathrm{HD}} \approx 260 \mathrm{fs}$, of screened longitudinal optical (LO) phonons expected in the heavily doped QW (54). The multiple factor 3 in $\tau_{\mathrm{e} \text {-ph }} \approx 3 \times \tau_{\mathrm{LO}, \mathrm{HD}}$ considers typical number of LO-phonons of energy $\hbar \omega_{\mathrm{LO}}$ $=36 \mathrm{meV}$ to be emitted for thermalizing hot electrons of typical mean energy $\left\langle\varepsilon_{\Gamma}\right\rangle=$ $80 \sim 110 \mathrm{meV}(21)$ in the $\Gamma$ valley. 


\section{S3. Simulation calculation of $\underline{\boldsymbol{E}}, \underline{T_{\mathrm{L}}} \underline{\text { and }} \underline{T_{\mathrm{e}}}$}

Simulation calculation is carried out by using a commercial software, COMSOL, based on a two-temperature model described in Ref. (36). Simplified assumption of linear transport due to single type of carriers is applied, so that neither the $\Gamma \rightarrow \mathrm{L}$ intervalley electron transfer nor any kind of conductivity variation is supposed. (The density and the mobility in the low-field limit at $300 \mathrm{~K}$ are used for simulation.)

Physical structure of the model is shown in fig. S4, where the electron- and the lattice-systems in the QW are represented by physically separated two layers, A and B, of different temperatures. Layer A represents the electron subsystem, which is characterized by the density $n_{2 D}=1.1 \times 10^{17} \mathrm{~m}^{-2} \quad\left(n_{3 D}=3.1 \times 10^{24} \mathrm{~m}^{-3}\right)$, the mobility $\mu=0.167 \mathrm{~m}^{2} / V \mathrm{~s}$, the electrical conductivity $\sigma=8.8 \times 10^{4} \mathrm{~S} / \mathrm{m}$, the electron thermal conductivity $\kappa_{\mathrm{e}}=0.1 \mathrm{~W} /(\mathrm{mK})$ and the electron specific heat $C_{\mathrm{e}}=6.5 \times 10$ $J /\left(\mathrm{Km}^{3}\right)$. Layer B represents GaAs lattice characterized by the thermal conductivity $\kappa \mathrm{L}=50 \mathrm{~W} /(\mathrm{mK})$ and the specific heat $C_{\mathrm{e}}=1.7 \times 10^{6} \mathrm{~J} /\left(\mathrm{Km}^{3}\right)$. Conduction electrons gain energy from the electric field $\boldsymbol{E}(\boldsymbol{r})$ through $p(\boldsymbol{r})=\boldsymbol{j}(\boldsymbol{r}) \cdot \boldsymbol{E}(\boldsymbol{r})=\sigma \boldsymbol{E}^{2}$. The gained energy is, in turn, either released to the lattice via electron phonon interaction or diffuses to the surrounding electrons through electron heat conduction. The energy released to the lattice is given by $\left(T_{\mathrm{e}}-T_{\mathrm{L}}\right) C_{\mathrm{e}} / \tau_{\mathrm{e}-\mathrm{ph}}$, where $\tau_{\mathrm{e}-\mathrm{ph}}$ is the electron-phonon energy relaxation time $\tau_{\mathrm{e}-\mathrm{ph}}$ and $T_{\mathrm{e}}=T_{\mathrm{e}}(\mathrm{x}, \mathrm{y})$ and $T_{\mathrm{L}}(\mathrm{x}, \mathrm{y})$ are, respectively, the local temperatures of layers $\mathrm{A}$ and $\mathrm{B}$. Here, the electron-phonon interaction is mimicked by the effective interface thermal resistance $h_{\mathrm{e}-\mathrm{ph}}=\tau_{\mathrm{e}-\mathrm{ph}} / C_{\mathrm{e}}=4.3 \times 10^{-7} \mathrm{Km}^{2} / \mathrm{W}$ between the two layers. Lateral diffusion of the electron energy within the electron system (Layer A) through the electron thermal conduction is described by $-\kappa_{\mathrm{e}} \nabla T_{\mathrm{e}}$. After released to the lattice, the heat spreads laterally within the lattice (Layer B) through $-\kappa_{\mathrm{L}} \nabla T_{\mathrm{L}}$. The heat is eventually transferred to the substrate (layer $\mathrm{C}$ ) and absorbed by the heat sink.

As shown in fig. S5, electric field $\boldsymbol{E}$ is sharply intensified at the corners reaching roughly $E_{\text {corner }} \sim 20 \mathrm{kV} / \mathrm{cm}$ at $V_{\mathrm{b}}=10.0 \mathrm{~V}$ (the current crowding effect). The average intensity of $\boldsymbol{E}$ in the channel is roughly $E_{\mathrm{av}} \approx 2.6 \mathrm{kV} / \mathrm{cm}\left(V_{\mathrm{b}}=10.0 \mathrm{~V}\right)$ in agreement with the value derived from the average total length of the $\mathrm{N}$-shaped channel. As is evident in fig. S5 B, $\boldsymbol{E}$ is reduced below $E_{\mathrm{av}} \approx 2.6 \mathrm{kV} / \mathrm{cm}$ in the outer region of the corners (further than $600 \mathrm{~nm}$ away from the corners). It is noted in fig. S5 B that $\boldsymbol{E}$ is slightly enhanced in the intermediate region connecting the two corners, along which elongated HED develops (Fig. 2 D).

Simulated profile of $T_{\mathrm{L}}$ and that of $T_{\mathrm{e}}$ for $V_{\mathrm{d}}=10 \mathrm{~V}$ (Fig. $3 \mathrm{D}$ ) are replotted in figs. S6 A. One-dimensional profiles of $T_{\mathrm{L}}$ and $T_{\mathrm{e}}$ in fig. $\mathrm{S} 6 \mathrm{~B}$ elucidate that the $T_{\mathrm{L}}$-profile is broad while $T_{\mathrm{e}}$-profile exhibits sharp peaks at the corners. As shown in figs. $\mathrm{S} 6 \mathrm{C}$ the profile of sharp $T_{\mathrm{e}}$ peak is kept substantially unchanged with increasing $V_{\mathrm{b}}$ from $6.0 \mathrm{~V}$ up to $10 \mathrm{~V}$. The feature of the sharp $T_{\mathrm{e}}$-profile (replotted in fig. $\mathrm{S} 7 \mathrm{C}$ ) is in contrast to the experimentally obtained $T_{\mathrm{e}}$ profiles displayed in figs. $\mathrm{S} 7 \mathrm{~A}$ and $\mathrm{B}$.

S4. Hot Electron Domain (HED): High-electric-field domain or high-current filament?

It is well known that, in the regime of negative differential conductivity (NDC), a high-current filament or a high-electric field domain is spontaneously formed depending on whether the current-voltage characteristic is S-shaped or N-shaped (55). 
The transport in this work is quasi-linear, without any signature of NDC. The observed HED is a region of higher $T_{\mathrm{e}}$, which must be generated by higher energy gain from $\boldsymbol{E}$, $p(\boldsymbol{r})=j(\boldsymbol{r}) \boldsymbol{E}(\boldsymbol{r})=\sigma \boldsymbol{E}^{2}$, than in the surrounding region. If the conductivity $\sigma$ is a constant and $j$ is accurately proportional to $E, j$ and $E$ would be equally higher in the HED. This is not the case in the experiment, where $\sigma$ is a decreasing function of $\boldsymbol{E}$. It follows that $p(\boldsymbol{r})=\sigma(\boldsymbol{r}) \boldsymbol{E}(\boldsymbol{r})^{2}$ can be higher only when $\boldsymbol{E}(\boldsymbol{r})$ is higher, leading to the conclusion that the observed HED is a high- $E$ region, not a high-current region. (We mention that, in the opposite nonlinear condition where the resistivity $\rho$ is a decreasing function of $\boldsymbol{E}$ $(\boldsymbol{j})$, a high-current filament occurs because $p(\boldsymbol{r})=\rho(\boldsymbol{r}) j(\boldsymbol{r})^{2}$ can be larger only when $\boldsymbol{j}(\boldsymbol{r})$ is larger.)

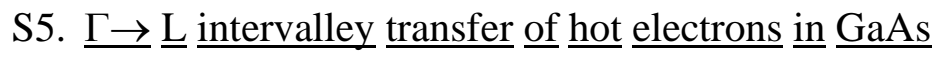

In GaAs the bottom of the conduction band lies in the isotropic $\Gamma$ valley, and the second local minima occurs in the satellite L-valleys - - - four equivalent valleys at $\mathrm{L}$ points - - - with a valley splitting energy $\Delta \varepsilon_{\Gamma L} \approx 250 \mathrm{meV}$ above the $\Gamma$-valley minimum (26). Whereas third local minimum occurs in the X-valleys with a splitting energy $\Delta \varepsilon \Gamma \mathrm{x} \approx 450 \mathrm{meV}$, electron transfer to the $\mathrm{X}$-valleys does not play substantial roles except in the range of extremely intense $\boldsymbol{E}$-field such that $E \gg 10 \mathrm{kV} / \mathrm{cm}$. Hot electrons accelerated in $\Gamma$ valley above the threshold energy $\Delta \varepsilon_{\Gamma L}$ (plus one LO-phonon energy $37 \mathrm{meV}$ ) transfer to the $\mathrm{L}$ valleys at an intervalley scattering rate $1 / \tau_{\Gamma \mathrm{L}} \approx 1 /(150 \sim 400 \mathrm{fs})$ $(23,24)$ [also see chapter 11 of Ref. (39)]. The anisotropic effective mass of L valley electrons is given by $\left(\mathrm{m}_{\mathrm{L}} / /, \mathrm{m}_{\mathrm{L}}^{\perp}, \mathrm{m}_{\mathrm{L}}^{\perp}\right)=\left(1.90 \mathrm{~m}_{0}, 0.075 \mathrm{~m}_{0}, 0.075 \mathrm{~m}_{0}\right)$ with the densityof-state effective mass $m_{\text {density }}^{L}=\left(\mathrm{g}^{2} \mathrm{~m}_{\mathrm{L}} / \mathrm{m}_{\mathrm{L}}{ }^{\perp} \mathrm{m}_{\mathrm{L}}{ }^{\perp}\right)^{1 / 3}=0.554 \mathrm{~m}_{0}$, where $\mathrm{g}=4$ is the number of equivalent valleys.

According to Monte Carlo simulation (17), the relative population of L-valley electrons, $n_{\mathrm{L}} / n$, starts to rapidly increase above $E \approx 2.5 \mathrm{kV} / \mathrm{cm}: \quad$ Letting $\left\{\mathrm{n}_{\Gamma} / \mathrm{n},\left\langle\varepsilon_{\Gamma}\right\rangle\right\}$

and $\left\{n_{L} / n,\left\langle\varepsilon_{L}>\right\}\right.$ be the relative populations and the average kinetic energies in respective valleys, and ignoring small electron population in $\mathrm{X}$ valleys, they are roughly estimated to vary as $\left\{\mathrm{n}_{\Gamma} / \mathrm{n},\left\langle\varepsilon_{\Gamma}>\right\} \approx\{96 \%, 80 \mathrm{meV}\} \rightarrow\{87.9 \%, 100 \mathrm{meV}\} \rightarrow\{65.3 \%\right.$, $120 \mathrm{meV}\} \rightarrow\{44.8 \%, 140 \mathrm{meV}\}$ and $\left\{\mathrm{n}_{\mathrm{L}} / \mathrm{n},\left\langle\varepsilon_{\mathrm{L}}\right\rangle\right\} \approx\{4 \%, 20 \mathrm{meV}\} \rightarrow\{12.1 \%$, $25 \mathrm{meV}\} \rightarrow\{34.7 \%, 30 \mathrm{meV}\} \rightarrow\{55.2 \%, 35 \mathrm{meV}\}$ as $\boldsymbol{E}$ increases as $E=2.6 \mathrm{kV} / \mathrm{cm} \rightarrow$ $3.0 \mathrm{kV} / \mathrm{cm} \rightarrow 4.0 \mathrm{kV} / \mathrm{cm} \mathrm{kV} / \mathrm{cm} \rightarrow 5.0 \mathrm{kV} / \mathrm{cm}$. With further increasing $\boldsymbol{E}$ above 5.0 $\mathrm{kV} / \mathrm{cm}, \mathrm{n}_{\mathrm{L}} / \mathrm{n}$ is nearly saturated at $\sim 65 \%$ and starts to decrease above $E \approx 10 \mathrm{kV} / \mathrm{cm}$ because the electron transfer to the $\mathrm{X}$ valleys comes to play a dominant role $(19,21$, 56).

S6. Derivation of $\underline{T_{\mathrm{e}}} \underline{\text { in }} \underline{\mathrm{SNoiM}}$

Effective temperature, $T_{\mathrm{e}}$ of hot electrons is estimated from the amplitude of signal, $V_{\text {sig }}$, of SNoiM without using any adjustable parameter. The physical implication of $V_{\text {sig }}$ 
and the procedure of deriving $T_{\mathrm{e}}$ are discussed in detail in Ref. (16) or in sections 5 and 7 of Supplementary Materials attached to Ref. (19).

The basic assumption is that $V_{\text {sig }}$ is proportional to the energy density of electromagnetic (EM) evanescent field generated by conduction electrons

$u_{\mathrm{n}}\left(T_{\mathrm{e}}\right)=\rho_{e}(\omega)\left[\hbar \omega /\left\{\exp \left(\hbar \omega / k_{\mathrm{B}} T_{e}\right)-1\right\}\right]$

at the probe-tip position (height $\mathrm{z}$ ) above the sample surface. Here $\rho_{\mathrm{e}}(\omega)$ is the electromagnetic local density of states (EM-LDOS) of the target, evaluated as a function of $\omega$ and $z$ by using the Fresnel reflection coefficients of the conduction electrons (57). (Small contribution from the lattice to the EM-LDOS is ignored.)

Absolute values of $T_{\mathrm{e}}$ are derived from the signal intensity, $\mathrm{S}(1)$, by calibrating with the experimental value obtained on a Au plate in thermal equilibrium at $T_{\text {Room }}=$ $300 \mathrm{~K}, \quad u_{\mathrm{Au}}\left(T_{\mathrm{Room}}\right)=\rho_{\mathrm{Au}}(\omega)\left[\hbar \omega /\left\{\exp \left(\hbar \omega / k_{\mathrm{B}} T_{\text {Room }}\right)-1\right\}\right], \quad$ through the relation

$u_{\mathrm{n}}\left(T_{\mathrm{e}}\right) / u_{\mathrm{Au}}\left(T_{\text {Room }}\right)=\left(\rho_{\mathrm{e}} / \rho_{\mathrm{Au}}\right) \times\left\{\exp \left(\hbar \omega / k_{\mathrm{B}} T_{\text {Room }}\right)-1\right\} /\left\{\exp \left(\hbar \omega / k_{\mathrm{B}} T_{\mathrm{e}}\right)-1\right\}$.

Since $\rho_{\mathrm{e}} / \rho_{\mathrm{Au}} \approx 10.0$ is theoretically known (16), $T_{\mathrm{e}}$ is derived without using any adjustable parameter.

We mention that relation $\mathrm{S}(1)$ is equivalent to the general formula of the spectral power density of thermally agitated current fluctuation (Nyquist noise) in a conductor of resistance $R$ at temperature $T$ (58)

$S_{\mathrm{I}}=4\left[\hbar \omega /\left\{\exp \left(\hbar \omega / k_{\mathrm{B}} T\right)-1\right\}\right] / R$,

when $T$ is replaced with $T_{\mathrm{e}}$. The signal of SNoiM, given by Relation $\mathrm{S}(1)$, is hence regarded as hot-electron Nyquist noise (or shot noise) generalized to the mode of EM evanescent field.

\section{S7. Physical implication of $\underline{T_{\mathrm{e}}} \underline{\text { and }} \underline{\text { intervalley noise }}$}

If conduction electrons consist of a single type of charge carriers, and if their distribution function is described by a displaced Maxwellian (or a Fermi function) with the effective electron temperature $T_{\mathrm{e}}{ }^{*}$, the quantity $T_{\mathrm{e}}$ derived in SNoiM is identical to $T_{\mathrm{e}}{ }^{*}$ representing the mean kinetic energy of electrons.

In this work, where two types of electrons are relevant (44) in a highly nonequilibrium condition, rigorous quantitative treatment of $T_{\mathrm{e}}$ is difficult. We simply assume that $T_{\mathrm{e}}$ derived in SNoiM is linked to the mean electron temperature through,

$$
\left\langle T_{\mathrm{e}}>=\left\{\mathrm{n}_{\Gamma}<\varepsilon_{\Gamma}>+\mathrm{n}_{\mathrm{L}}<\varepsilon_{\mathrm{L}}>\right\} /\left(\mathrm{n} k_{\mathrm{B}}\right) .\right.
$$

While this is a qualitatively justified assumption at present, quantitative accuracy is not guaranteed for the three unknown factors below. 
First, electromagnetic local density of states (EM-LDOSs) should be separately treated for $\Gamma$ - and L-valley electrons, $\rho_{e, \Gamma}(\omega)$ and $\rho_{e, L}(\omega)$, so that $\mathrm{S}(1)$ should be replaced with $u_{\mathrm{n}}\left(T_{\mathrm{e}}\right)=\rho_{e, \Gamma}(\omega)\left[\hbar \omega /\left\{\exp \left(\hbar \omega / k_{\mathrm{B}} T_{e, \Gamma}\right)-1\right\}\right] \quad+$ $\rho_{e, L}(\omega)\left[\hbar \omega /\left\{\exp \left(\hbar \omega / k_{\mathrm{B}} T_{e, L}\right)-1\right\}\right]$ with $T_{e, \Gamma}$ and $T_{e, L}$ representing the effective electron temperatures of the respective valleys. (Equation (2) implicitly corresponds to the non-trivial assumption $\rho_{e, \Gamma}(\omega) / \rho_{e, L}(\omega)=\mathrm{n}_{\Gamma} / \mathrm{n}_{\mathrm{L}}$.)

Secondly both of the distribution functions of $\Gamma$ - and L-valley electrons are known to deviate substantially from displaced Maxwellian or Fermi distributions (21), making quantitative accuracy of derived predictions unclear.

Finally, intervalley scattering gives rise to additional electrical noise to the heating effect (43-46). All the existing studies so far have been restricted to the lowfrequency limit noise $\left(\omega \tau_{\text {int }}<<1\right)$ with $\tau_{\text {int }}=150 \sim 400$ fs being the intervalley scattering time (45). By assuming the noise to arise from the fluctuation in the drift velocity, it is given by $(43,46)$,

$$
S_{\text {int }} \propto\left(\mathrm{n}_{\Gamma} \mathrm{n}_{\mathrm{L}} / \mathrm{n}\right)\left(\left\langle v_{\Gamma}\right\rangle_{\mathrm{d}}-\left\langle v_{\mathrm{L}}\right\rangle_{\mathrm{d}}\right)^{2}\left[\tau_{\text {int }} /\left\{1+\left(\omega \tau_{\text {int }}\right)^{2}\right\}\right], \quad \mathrm{S}(4)
$$

where $\left\langle v_{\Gamma}\right\rangle_{\mathrm{d}}$ and $\left\langle v_{\mathrm{L}}\right\rangle_{\mathrm{d}}$ are the drift velocities in the respective valleys. (Intravalley scattering rate is assumed to be much higher than the intervalley transition rate.) The intervalley noise would be negligibly small if Relation $S(4)$ is to the present work. However, $\mathrm{S}(4)$ is not applicable because ultra-high frequency noise $\left(\omega \tau_{\mathrm{int}}=20 \sim 53>\right.$ $1)$ is studied. The instantaneous velocity change caused by individual events of intervalley scattering, far larger than the drift velocity difference $\left\langle\boldsymbol{v}_{\Gamma}\right\rangle_{\mathrm{d}}-\left\langle\boldsymbol{v}_{\mathrm{L}}\right\rangle_{\mathrm{d}}$, is not averaged out during the short period $(2 \pi / \omega \approx 48 \mathrm{fs})$ of noise frequency and possibly contributes to the ultra-high frequency noise. We hence suppose that intervalley noise could provide substancial contribution in this work $\left(\omega \tau_{\mathrm{nnt}}>>1\right)$. Particularly, it may possibly account for the large increase from $T_{\mathrm{e}, \mathrm{out}} \approx 1,200 \mathrm{~K}$ to $T_{\mathrm{e}, \mathrm{HED}} \approx 1,700 \mathrm{~K}$, which cannot be quantitatively explained by Eq. (2). 
Figs. S1 to S7

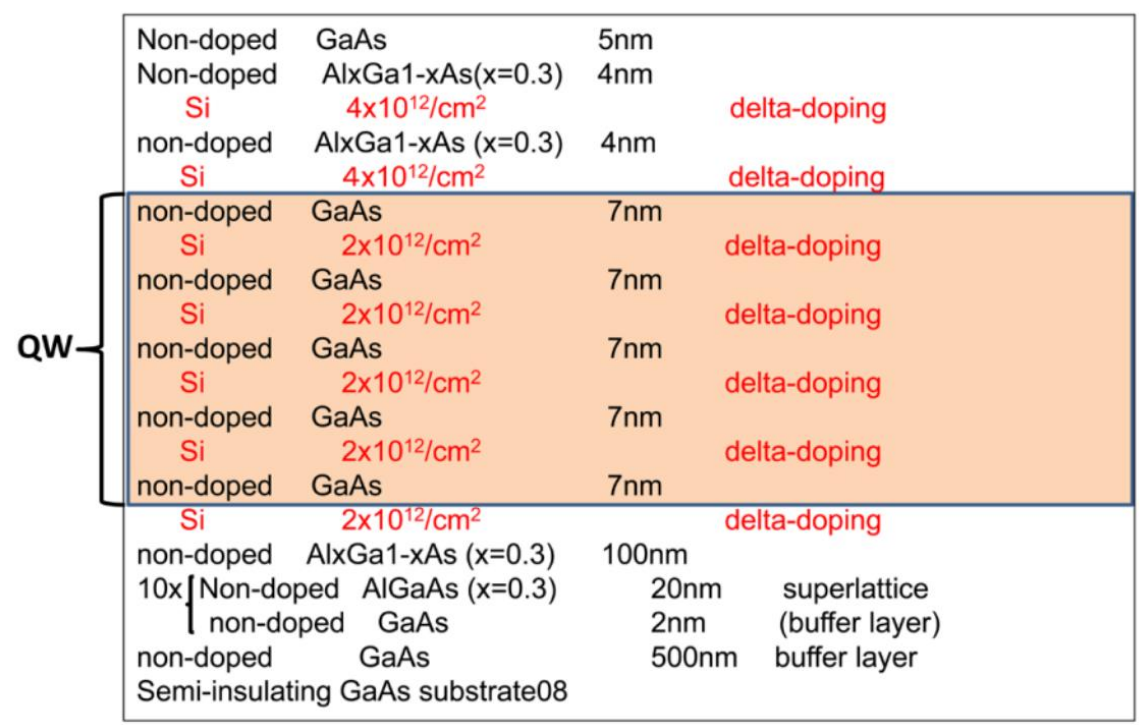

fig. S1. GaAs/AlGaAs quantum well structure 


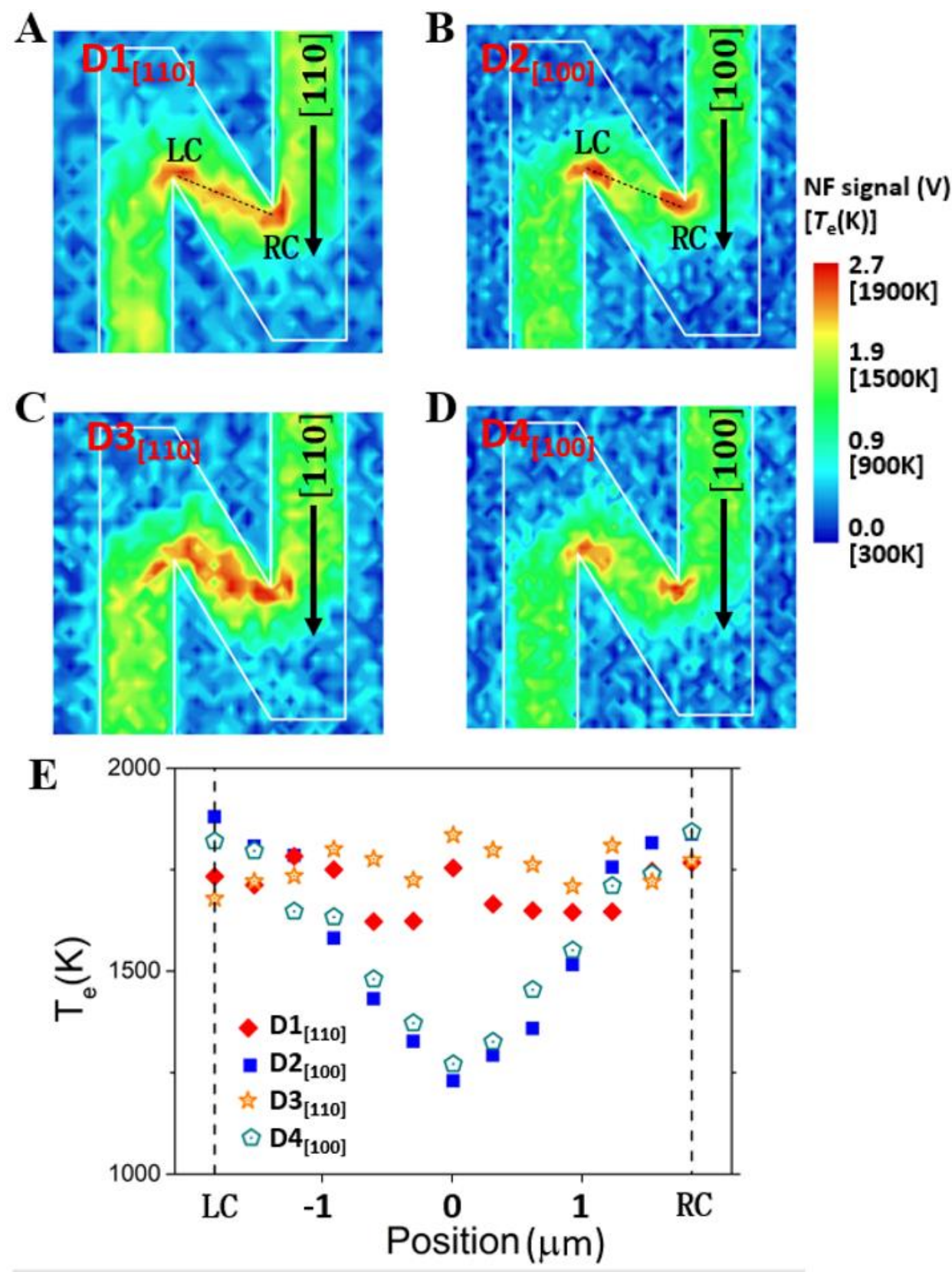

fig. S2. Crystallographic-orientation dependence: $T_{\mathrm{e}}$-profile at $\mathrm{V}_{\mathrm{b}}=+10 \mathrm{~V}$

A-D; Two-dimensional plots. E; One-dimensional profile along the line connecting the corners (indicated by a broken line in A and B). 

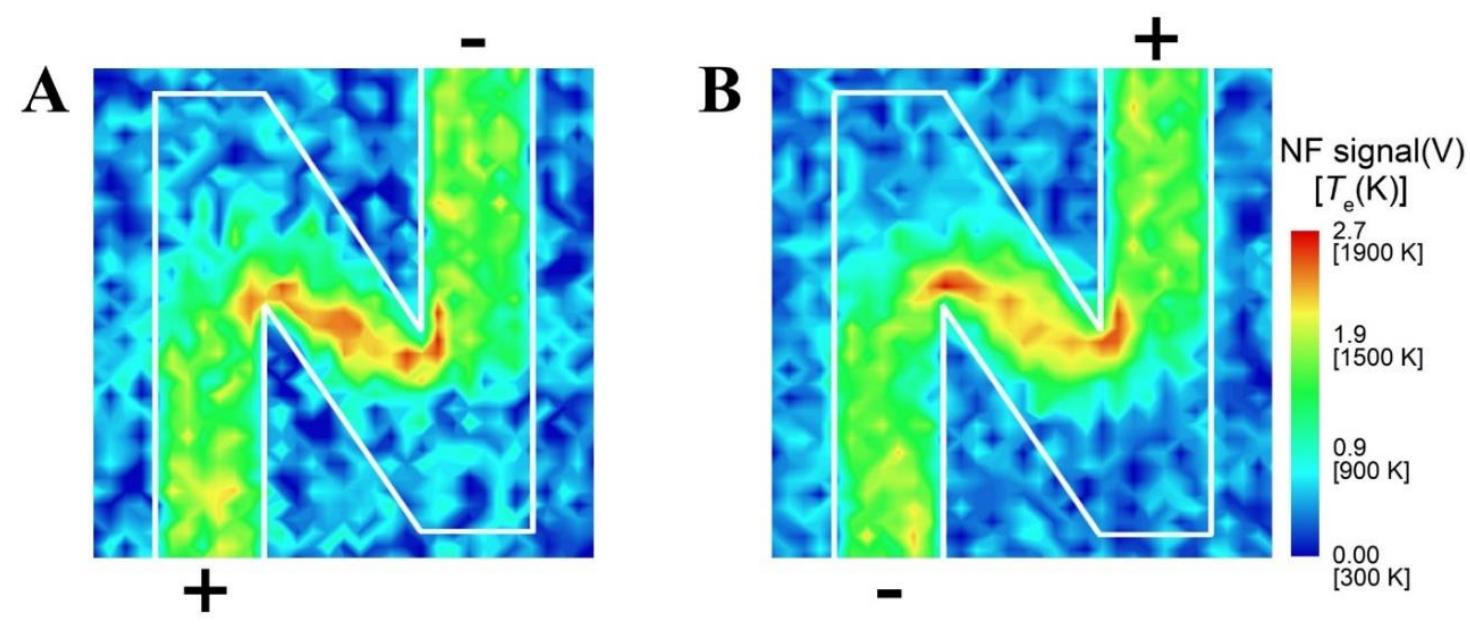

fig. S3. Bias-polarity dependence: $T_{\mathrm{e}}$-image in device $\mathrm{D} 1_{[110]}$ for $\mathrm{V}_{\mathrm{b}}= \pm 10 \mathrm{~V}$ 


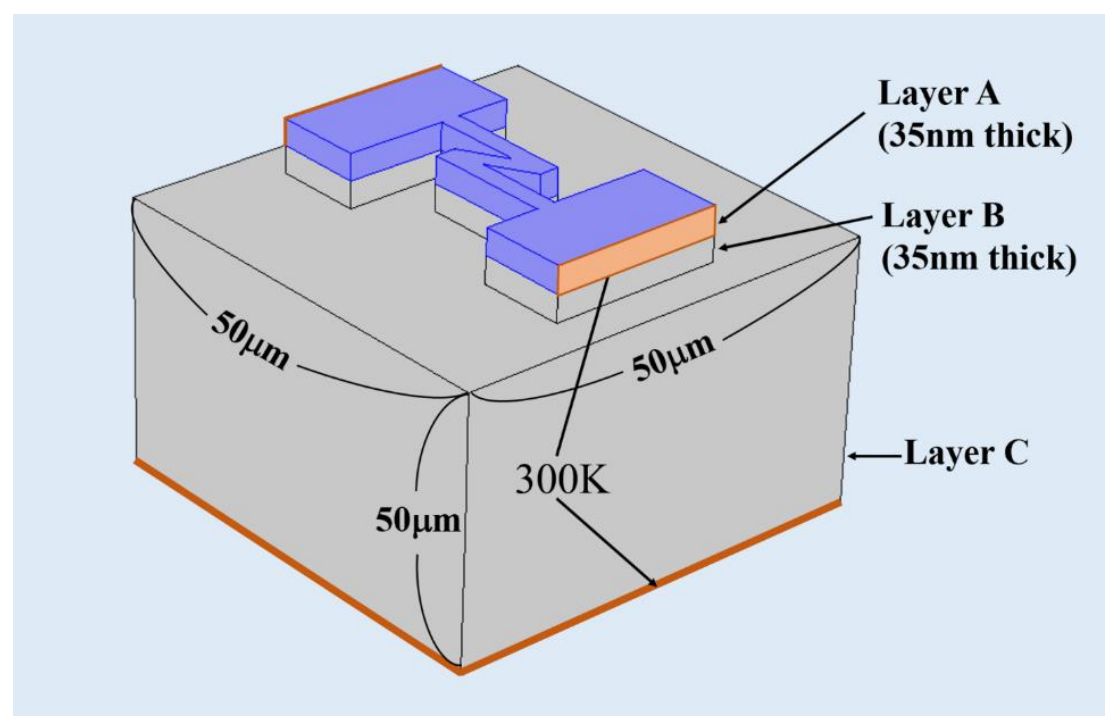

fig. S4. Physical model structure for simulation 
A

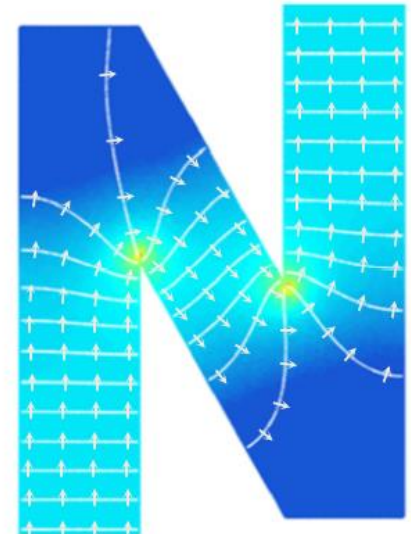

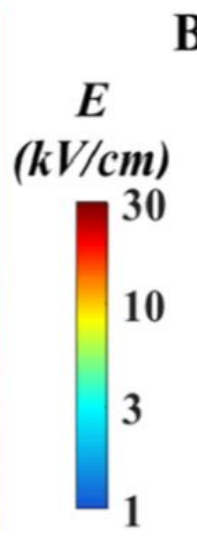

B

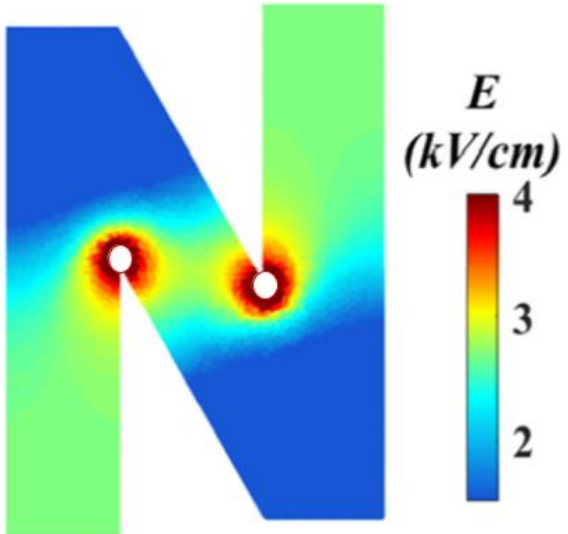

fig. S5. Simulation calculation of the electric field $E$ for $V_{\mathrm{b}}=10.0 \mathrm{~V}$

A: Logarithmic-scale color plot in the range $E=1.0 \sim 30 \mathrm{kV} / \mathrm{cm} . E_{\mathrm{av}} \approx 2.6 \mathrm{kV} / \mathrm{cm}$ is observed when averaged in the straight sections of the channel. The arrows indicate the directions of $\boldsymbol{E}$ and the current. White lines are equipotential lines at a step of $\Delta \mathrm{V}=0.15 \mathrm{~V}$.

B: Linear-scale color plot in the range $E=1.5 \sim 4.0 \mathrm{kV} / \mathrm{cm}$ for finer examination of the $\boldsymbol{E}$-field distribution. Hot spot regions of $E>4 \mathrm{kV} / \mathrm{cm}$ are indicated by the white areas. 

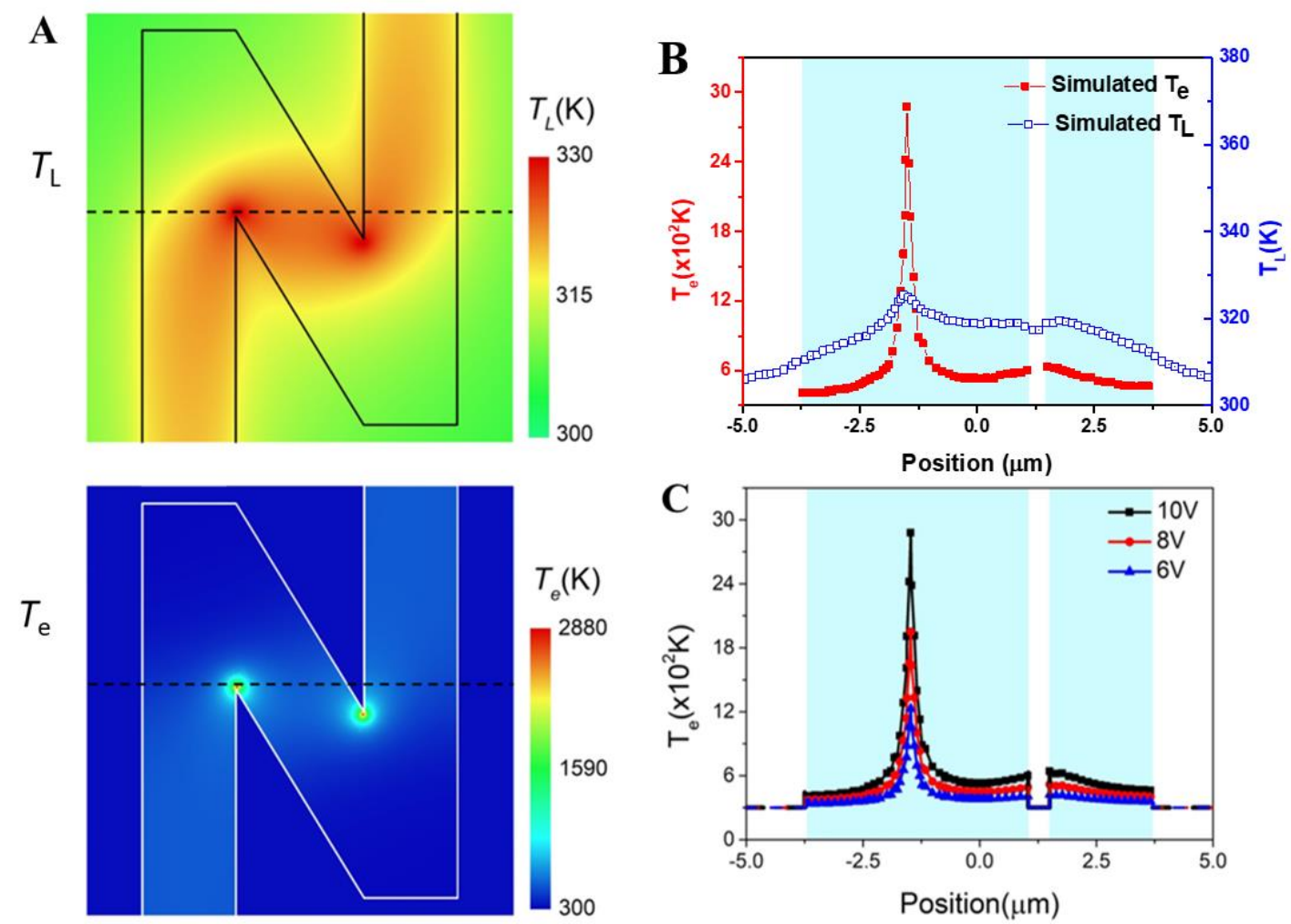

fig. S6. Simulation calculation: $T_{\mathrm{L}}$ and $T_{\mathrm{e}}$ profiles for $V_{b}=10 \mathrm{~V}$

(A) Replots of Fig.3 D. (B) One-dimensional profiles of $\mathrm{T}_{\mathrm{L}}$ and $\mathrm{T}_{\mathrm{e}}\left(V_{\mathrm{b}}=10 \mathrm{~V}\right)$ along the broken lines shown in (A). (D) One-dimensional profiles of $\mathrm{T}_{\mathrm{e}}$ at $V_{b}=6.0$, 8.0 and $10 \mathrm{~V}$. 
A
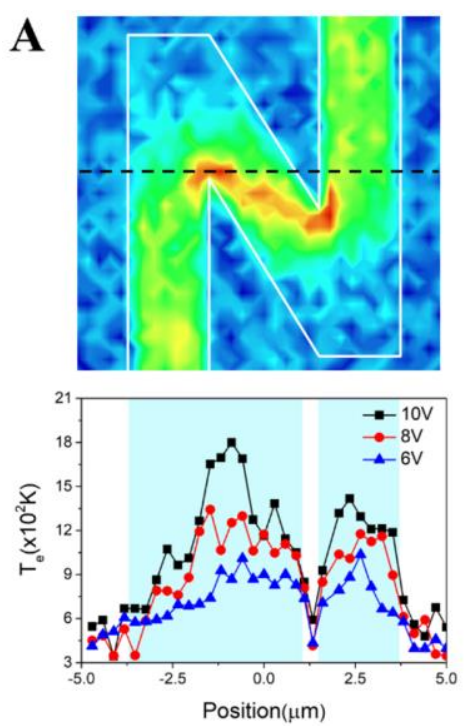

B
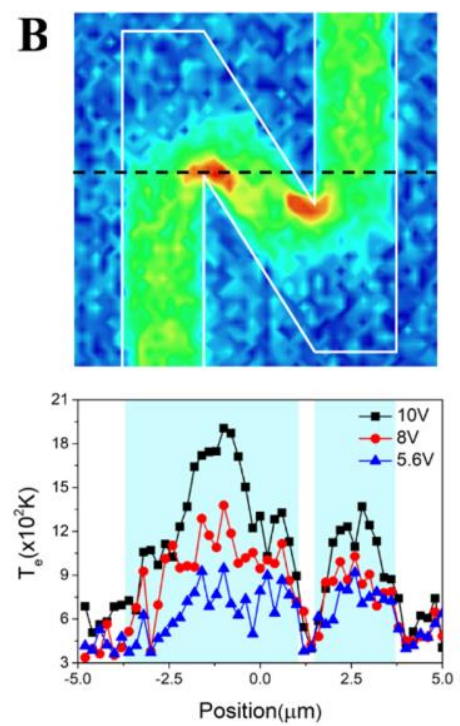

C
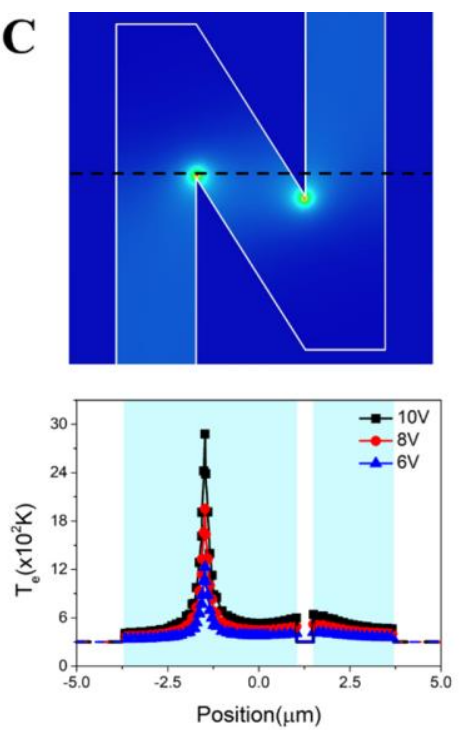

fig. S7. Comparison between experiments and simulation

A: D1 [110], B: D2 ${ }_{[100]}, \mathrm{C}:$ Simulation. The upper panel shows two-dimensional images for $V_{\mathrm{b}}=10 \mathrm{~V}$. The lower panel shows one-dimensional profiles along the lines marked in the upper panel for $V_{\mathrm{b}}=6.0,8.0$ and $10 \mathrm{~V}$. The hot-spot at the corner is sharp in the simulation but much broader in the experiments. 


\section{References (54 to 58)}

54. I. Ezhov, C. Jirauschek, Influence of screening on longitudinal-optical phonon scattering in quantum cascade lasers. J. Appl. Phys. 119, 033102 (2016).

55. J.Peinke et al., Spatio-temporal correlations in semiconductors, in Spontaneous Formation of Space-Time Structures and Criticality (ed. T.Riste and D.Sherrington, NATO ASI Series, Springer Science+Business Media,B.V, 1991) pp.145.

56. V. Aninkevičius et al., $\Gamma-\mathrm{X}$ intervalley-scattering time constant for GaAs estimated from hot-electron noise spectroscopy data. Phys. Rev. B 53, 6893 (1996).

57. K. Joulain et al., Surface electromagnetic waves thermally excited: Radiative heat transfer, coherence properties and Casimir forces revisited in the near field. Surf. Sci. Rep. 57, 59-112 (2005).

58. H. Nyquist, Thermal agitation of electric charge in conductors. Phys. Rev. 32, 110113 (1928). 